JOURNAL OF SYNCHROTRON RADIATION

ISSN $1600-5775$

Received 1 February 2018

Accepted 8 April 2018

Edited by M. Yamamoto, RIKEN SPring-8

Center, Japan

Keywords: synchrotron; bending magnets; Doppler; relativity; $\mathrm{X}$-rays.

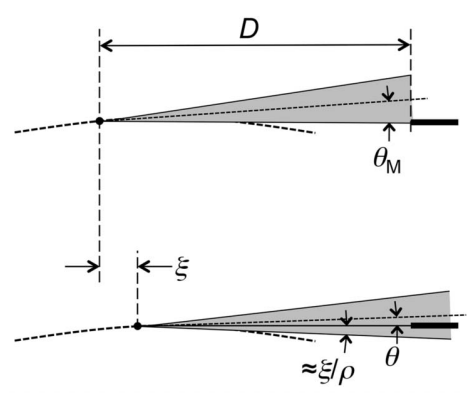

C 2018 International Union of Crystallography

\section{The simple physics of the bending magnet spectrum}

\author{
Giorgio Margaritondo*
}

Ecole Polytechnique Fédérale de Lausanne (EPFL), EPFL SB-DO, PH A1 365 (Bâtiment PH), Station 3, CH-1025 Lausanne, Switzerland. *Correspondence e-mail: giorgio.margaritondo@epfl.ch

A new elementary model of the bending magnet synchrotron radiation is presented, with minimal mathematical formalism. The model explains features not justified by other simplified approaches; in particular, it brings to light the key role of the directional Doppler effect.

1. Introduction: understanding the physics

A communication problem has affected synchrotron radiation during all six decades of its history. Initially, high-level theorists (Pomeranchuk, 1939; Iwanenko \& Pomeranchuk, 1944; Artsimivich \& Pomeranchuk, 1946; Schwinger, 1949) treated it specifically for accelerator experts: their mathematical formalism was quite complicated and the underlying physics was not easy to grasp. By contrast, in the following decades the synchrotron user community became multidisciplinary, with many of its members finding it difficult to deal with the original theories, sometimes missing excellent research opportunities. Alternate, simplified models were proposed (Attwood, 2007; Margaritondo, 1988, 2002; Mobilio et al., 2015; Margaritondo \& Rebernik Ribic, 2011; Rebernik Ribic \& Margaritondo, 2012) focusing on the physics background and minimizing formalism. But the results are not yet satisfactory. Recently, for example, it was argued that even the relativistic foundations of synchrotron emission are often misrepresented (Margaritondo \& Rafelski, 2017).

As part of an effort to develop better, easily understandable synchrotron models, we deal here with the spectral distribution of bending magnet radiation. We show that its previous simplified models are not entirely adequate: they do not explain some basic features and do not fully account for the role of the Doppler effect. An alternate simplified approach is proposed, tackling these problems and bringing to light (no pun intended!) the essential physics foundations.

\section{Previous models and their limitations}

Full theories of bending magnet emission (e.g. Mobilio et al., 2015) use the Liénard-Wiechert field retarded-potential framework. The results are expressed in terms of modified Bessel functions and can be numerically evaluated. An online engine is available to perform such calculations for any selected set of parameters (http://henke.lbl.gov/optical_ constants/bend2.html). Still, these formal or practical instruments suffer from the problems of difficult comprehension when used by non-physicists and non-specialists in general.

A simplified model of the bending magnet spectrum can start from the classical (non-relativistic) Newton's law for the cyclotron motion of an electron subject to a constant magnetic 
field of strength $B$, moving with speed $v$ along a circular segment in a plane perpendicular to the field,

$$
\frac{e v B}{m}=a,
$$

where $e$ and $m$ are the electron charge and (rest) mass, $e v B$ is the magnitude of the magnetic (transverse) force, and $a$ is the acceleration magnitude. During an infinitesimal time $\mathrm{d} t$, the speed changes by $\mathrm{d} v=a \mathrm{~d} t$ in the transverse direction, corresponding to an angle $\mathrm{d} v / v=a \mathrm{~d} t / v$ and to an angular speed

$$
\omega_{0}=\frac{(a \mathrm{~d} t / v)}{\mathrm{d} t}=\frac{a}{v}=\frac{e B}{m},
$$

which is the so-called 'cyclotron' (angular) frequency. The centripetally accelerated charged electron emits radiation centered around the wavelength

$$
\lambda_{0}=\frac{2 \pi c}{\omega_{0}}=\frac{2 \pi c m}{e B} .
$$

When $v$ approaches the speed of light $c$, relativity significantly affects equation (3). In particular, the wavelength $\lambda_{0}$ in the laboratory reference frame $R$ is increasingly different from the wavelength $\lambda_{0}^{\prime}$ in the 'electron' frame $R^{\prime}$.

Specifically, $R^{\prime}$ is the inertial frame whose constant velocity instantaneously coincides with the electron velocity. The electron speed is zero in $R^{\prime}$, but its (transverse) acceleration magnitude $a^{\prime}$ is not. To calculate $\lambda_{0}^{\prime}$, we must compare $a$ and $a^{\prime}$. The transverse coordinates are relativistic invariants, thus these accelerations transform like the reciprocal square of the time: $a=a^{\prime} / \gamma^{2}$, where $\gamma$ is the usual factor

$$
\gamma=\frac{1}{\left(1-\beta^{2}\right)^{1 / 2}}=\frac{1}{\left(1-v^{2} / c^{2}\right)^{1 / 2}} .
$$

In the $R$-frame, $a$ is now given by the relativistic version of Newton's law [equation (1), but with $\gamma m$ instead of $m$ ],

$$
\frac{e v B}{\gamma m}=a,
$$

therefore

$$
\frac{\gamma e v B}{m}=a^{\prime},
$$

(the relativistic Newton's law in the $R^{\prime}$-frame, where mass = $m$ ). By replacing $a$ with $a^{\prime}$ in equation (2), we obtain the frequency $\omega_{0}^{\prime}$ in $R^{\prime}$ and the wavelength

$$
\lambda_{0}^{\prime}=\frac{2 \pi c m}{\gamma e B} .
$$

However, $\lambda_{0}^{\prime}$ is not the detected wavelength $\lambda_{0}$ in the laboratory $R$-frame, due to the Doppler shift caused by electron motion. For acoustic waves, the Doppler shift is a phenomenon of everyday life, observed when the source and the observer move with respect to each other, e.g. for the siren of a passing ambulance. For electromagnetic waves, it is a relativistic effect: the Lorentz transformations imply (Rafelski, 2017) that the wavelength is multiplied, in the longitudinal direction, by the factor

$$
\left(\frac{1-\beta}{1+\beta}\right)^{1 / 2}=\frac{\left(1-\beta^{2}\right)^{1 / 2}}{1+\beta}=\frac{1}{\gamma(1+\beta)},
$$

which for $v \simeq c$ and $\beta \simeq 1$ becomes $\sim 1 /(2 \gamma)$, so that the detected bending magnet wavelength in the laboratory $R$ frame is

$$
\lambda_{0} \simeq \frac{\lambda_{0}^{\prime}}{2 \gamma}=\frac{2 \pi c m}{2 \gamma^{2} e B},
$$

a value close to the 'critical wavelength' $4 \pi \mathrm{cm} /\left(3 \gamma^{2} e B\right)$ derived from full synchrotron radiation theories (Mobilio et al., 2015).

The value $\lambda_{0}$ is at the center of a very broad range of emitted wavelengths. The previous simplified approaches (Margaritondo, 2002; Attwood, 2007) approximately modeled this spectrum as a broad peak centered near $\lambda_{0}$ and with a bandwidth $\Delta \lambda$. The bandwidth $\Delta \lambda$ was estimated by calculating the duration $\Delta t$ of the radiation pulse reaching a pointlike detector and by using the Fourier transform properties to obtain $\Delta \omega$, the frequency bandwidth, and from it $\Delta \lambda$.

The result is a rather large bandwidth,

$$
\Delta \lambda \simeq \frac{2 \pi c m}{\ln 2 \gamma^{2} e B}=\frac{2}{\ln 2} \lambda_{0} .
$$

Note, by the way, that this approach could also be used to roughly estimate the peak wavelength without the steps leading from equation (1) to equation (9), by simply assuming that $\lambda_{0} \simeq \Delta \lambda / 2$, and obtaining the same result as equation (9) except for the factor $1 / \ln 2$.

Is it realistic, however, to consider the emission spectrum simply as a broad peak? This picture is not immediately reminiscent of the 'universal' bending magnet spectral distribution (Mobilio et al., 2015). However, the conventional way to show this distribution uses log-log plots, and this may confuse the comparison.

The problem, though, is not just a question of plots. The shortcomings of the previous simple models become evident if one analyzes the linear-linear graphs of the spectra calculated from full synchrotron theories (see, for example, Fig. 1).

At first glance, this curve does look like a broad peak with a maximum not too far from the numerical value of $\lambda_{0}$ from equation (6), $\lambda_{0} \simeq 1.4 \mathrm{~nm}$. But there is a key additional feature: a marked asymmetry of the peak, with a sharp cut on the short-wavelength side and an extended tail at long wavelengths. This is a very important property, limiting the emission in the X-ray range (which is the most useful), whereas it extends it to the infrared range, paving the way to the corresponding specialized applications.

\section{Our model: qualitative version}

Previous simplified models do not explicitly explain the asymmetry of Fig. 1. Here, we argue that its primary cause is the directional dependence of the Doppler shift, a key but overlooked factor. In the case of acoustic waves, this directional effect is again a fact of everyday life: for example, the frequency change of a train noise caused by its motion is more noticeable if the observer is close to the tracks. Likewise, the 


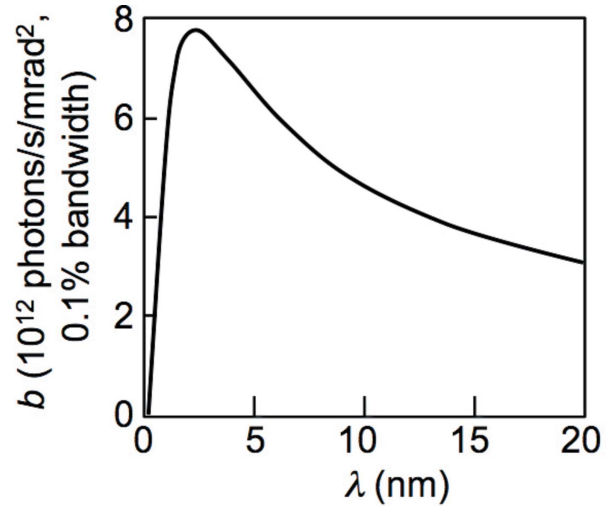

Figure 1

Spectral brightness $b$ of a bending magnet with $B=1 \mathrm{~T}$, for an electron energy of $1 \mathrm{GeV}\left(\gamma \simeq 1.96 \times 10^{3}\right)$ and for an electron beam current of $400 \mathrm{~mA}$. The curve was obtained with the calculation engine of the website http://henke.lbl.gov/optical_constants/bend2.html.

relativistic Doppler shift of the electromagnetic waves changes with $\theta$, the angle between the (longitudinal) source motion and the source-observer line.

Relativity predicts the general form of the Doppler factor (Rafelski, 2017),

$$
\gamma(1-\beta \cos \theta)
$$

which for $\theta=0$ becomes equal to equation (8). This directional factor has an important impact on the bending magnet spectrum. Consider indeed Fig. 2(a), showing the narrow emission 'cone' of an electron passing through a bending magnet, when it starts to illuminate a point-like detector. The detected wavelength at this time is not $\lambda_{0}$ as given by equation (9), since this equation was derived using the longitudinal Doppler shift. In Fig. 2(a), instead, the line of sight leading to the detector is at an angle $\theta_{\mathrm{M}}$ with respect to the longitudinal direction.

(a)

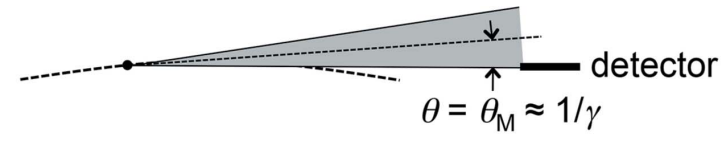

(b)

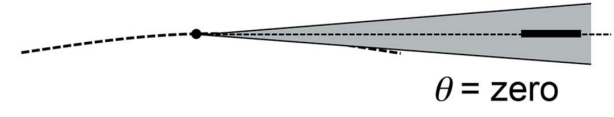

(c)

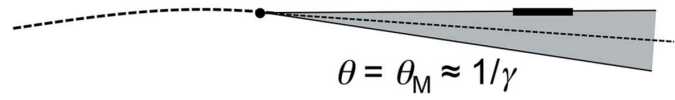

Figure 2

An electron passes through a bending magnet and its narrow synchrotron 'cone' of width $\sim 2 / \gamma$ sweeps through a point-like detector. (a) When the detection starts, the electron-detector line of sight is at an angle $\theta=\theta_{\mathrm{M}} \simeq$ $1 / \gamma$ from the longitudinal direction. Then $(b) \theta$ decreases to zero, and afterwards $(c)$ it increases again to $\theta_{\mathrm{M}}$.
The directional Doppler factor of equation (11) increases with $\theta$, so the corresponding wavelength $\lambda\left(\theta_{\mathbf{M}}\right)$ is larger than $\lambda_{0}$ : we shall see indeed that $\lambda\left(\theta_{\mathrm{M}}\right) \simeq 2 \lambda_{0}$. This is the detected wavelength immediately after the emission cone starts illuminating the detector (Fig. 2a). Afterwards, as the electron travels along its trajectory, the cone rotates until the detected radiation corresponds to the longitudinal Doppler shift, i.e. to $\lambda_{0}$ (see Fig. $2 b$ ). Then, the electron motion and the corresponding cone rotation bring the direction of detection off the longitudinal direction: the detected wavelength increases until it reaches again the maximum value, $\sim 2 \lambda_{0}$ (Fig. $2 c$ ).

This mechanism qualitatively explains the asymmetry of the emission spectrum of Fig. 1. In simple terms, due to the directional dependence of the Doppler shift the detector spends a lot of time revealing wavelengths longer than $\lambda_{0}$.

\section{Formal version}

Our theoretical treatment of the above mechanism starts with the calculation of $\theta_{\mathrm{M}}$, the half-width of the emission cone, which can be estimated by considering a photon emitted in the electron frame $R^{\prime}$ along the extreme (transverse) direction. The photon velocity components are $c_{x}^{\prime}=0$ in the longitudinal direction $x^{\prime}$, and $c_{y}^{\prime}=c$ in the transverse direction $y^{\prime}$. The relativistic velocity transformations (Rafelski, 2017) from $R^{\prime}$ to $R$ give

$$
c_{x}=\frac{c_{x}^{\prime}+v}{1+c_{x}^{\prime} v / c^{2}}=v=\beta c, \quad c_{y}=\frac{c_{y}^{\prime}}{\gamma}=\frac{c}{\gamma}
$$

[note that $c_{x}^{2}+c_{y}^{2}=\beta^{2} c^{2}+c^{2} / \gamma^{2}=\left(\beta^{2}+1-\beta^{2}\right) c^{2}=c^{2}$ as required by relativity]. The corresponding maximum angle $\theta_{\mathrm{M}}$ in the $R$-frame is equal to $\tan ^{-1}\left(c_{y} / c_{x}\right)$. Since this angle is very small because of the factor $1 / \gamma$ in equation $(8), \tan ^{-1}\left(c_{y} / c_{x}\right) \simeq$ $c_{y} / c_{x}$, and

$$
\theta_{\mathrm{M}}=\frac{c_{y}}{c_{x}}=\frac{1}{\beta \gamma} \simeq \frac{1}{\gamma},
$$

a well known result of all synchrotron theories.

Next, we must calculate the wavelength Doppler shift factor for $\theta_{\mathrm{M}}$. For small angles $\theta$, and assuming $\beta=v / c \simeq 1$, the Doppler factor of equation (11) becomes

$$
\begin{aligned}
\gamma(1-\beta \cos \theta) & \simeq \gamma\left[1-\beta\left(1-\frac{\theta^{2}}{2}\right)\right] \\
& =\gamma\left[(1-\beta)+\beta \frac{\theta^{2}}{2}\right] \\
& =\gamma(1-\beta)\left[1+\beta \frac{\theta^{2}}{2(1-\beta)}\right] \\
& =\gamma \frac{\left(1-\beta^{2}\right)}{1+\beta}\left[1+\beta \frac{\theta^{2}(1+\beta)}{2\left(1-\beta^{2}\right)}\right] \\
& \simeq \gamma \frac{1}{2 \gamma^{2}}\left[1+\beta \frac{2 \theta^{2} \gamma^{2}}{2}\right] \\
& \simeq \frac{1}{2 \gamma}\left(1+\theta^{2} \gamma^{2}\right) .
\end{aligned}
$$


If $\theta=\theta_{\mathbf{M}} \simeq 1 / \gamma$ (Fig. $2 a$ ), equation (14) gives

$$
\frac{1}{2 \gamma}\left(1+\theta_{\mathrm{M}}^{2} \gamma^{2}\right) \simeq \frac{1}{\gamma}
$$

the wavelength $\lambda\left(\theta_{M}\right)$ can be derived from equation (9) by replacing the factor $1 /(2 \gamma)$ with $1 / \gamma$,

$$
\lambda\left(\theta_{\mathrm{M}}\right) \simeq 2 \lambda_{0},
$$

which is the value we used for the qualitative description of the previous section. In general, the detected wavelength $\lambda(\theta)$ for an angle $\theta$ can be derived by replacing the Doppler factor $1 /(2 \gamma)$ in equation (9) with that of equation (14),

$$
\lambda(\theta) \simeq \frac{\lambda_{0}^{\prime}}{2 \gamma}\left(1+\theta^{2} \gamma^{2}\right)=\lambda_{0}\left(1+\theta^{2} \gamma^{2}\right)
$$

Next, we must link the wavelength to the detection time. Referring to Fig. 3(a), the detection starts at a time $D / c$ after the corresponding emission, where $D$ is the initial electrondetector distance. In Fig. 3(b), the electron-detector distance has decreased to $\sim(D-\xi)$, thus the detection takes place at a time $\sim(D-\xi) / c$ after the emission. The distance in time between the emissions of Fig. 3(a) and 3(b) is $\sim \xi / v$. Thus, the detection of Fig. 3(b) takes place at the time (measured from the beginning of the detection)

$$
\tau \simeq \frac{\xi}{v}+\frac{D-\xi}{c}-\frac{D}{c}=\xi\left(\frac{1}{v}-\frac{1}{c}\right)=\frac{\xi}{v}(1-\beta) .
$$

Therefore,

$$
\xi \simeq \frac{\tau v}{1-\beta}
$$

Using the trajectory curvature radius $\rho, \xi$ corresponds to a cone rotation angle $\xi / \rho$ and therefore to the following angle between the longitudinal direction and the detection direction,

$$
\theta=\theta_{\mathrm{M}}-\frac{\xi}{\rho} \simeq \frac{1}{\gamma}-\frac{\tau \nu}{(1-\beta) \rho}=\frac{1}{\gamma}-\Omega \tau
$$

where

(a)

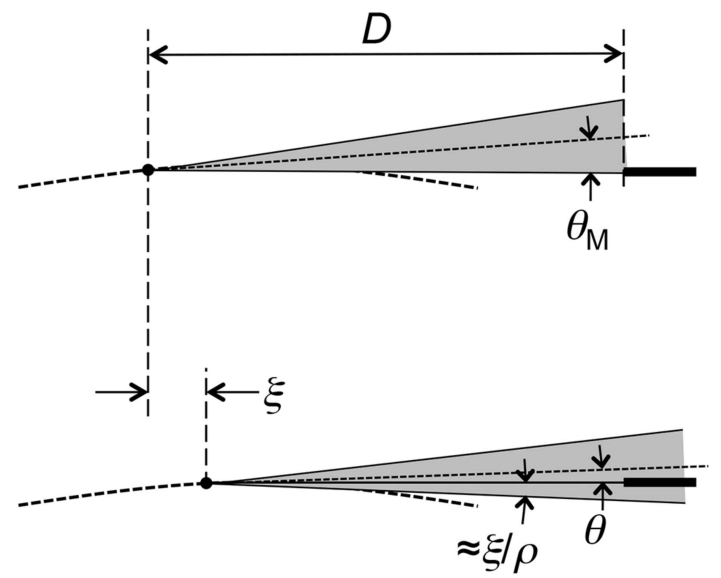

Figure 3

The emitting electron of Fig. 2, shown $(a)$ for the start of the detection and $(b)$ after it has traveled over a distance $\sim \xi$.

$$
\Omega=\frac{v}{(1-\beta) \rho} .
$$

Thus, the detected wavelength at the time $\tau$ is, according to equation (17),

$$
\begin{aligned}
\lambda(\tau) & \simeq \lambda_{0}\left(1+\theta^{2} \gamma^{2}\right) \\
& =\lambda_{0}\left[1+\gamma^{2}\left(\frac{1}{\gamma}-\Omega \tau\right)^{2}\right] \\
& =\lambda_{0}\left[1+(1-\gamma \Omega \tau)^{2}\right],
\end{aligned}
$$

which gives

$$
\frac{\lambda}{\lambda_{0}}=1+(1-\gamma \Omega \tau)^{2}
$$

The plot of this time-dependent wavelength is shown in Fig. 4. We see that, except in the middle of the detection-time interval, the wavelength is longer than $\lambda_{0}$, justifying the asymmetry in Fig. 1 and corroborating our qualitative arguments.

To derive the spectral intensity distribution, we obtain from equation (23),

$$
\tau=\frac{ \pm\left[\left(\lambda / \lambda_{0}\right)-1\right]^{1 / 2}+1}{\gamma \Omega}
$$

so that

$$
\frac{\mathrm{d} \tau}{\mathrm{d} \lambda}= \pm \frac{1}{2 \gamma \Omega \lambda_{0}} \frac{1}{\left[\left(\lambda / \lambda_{0}\right)-1\right]^{1 / 2}} .
$$

Assume now that $G(\lambda) \mathrm{d} \lambda$ is the detected signal in the wavelength interval $\mathrm{d} \lambda$. Energy conservation requires

$$
G(\lambda) \mathrm{d} \lambda=P(\tau) \mathrm{d} \tau,
$$

where $P(\tau)$ is the detected power at the time $\tau$. For simplicity, we will assume that $P(\tau)$ is constant and equal to $P_{0}$ during the entire detection period, i.e. that the angular power distribution

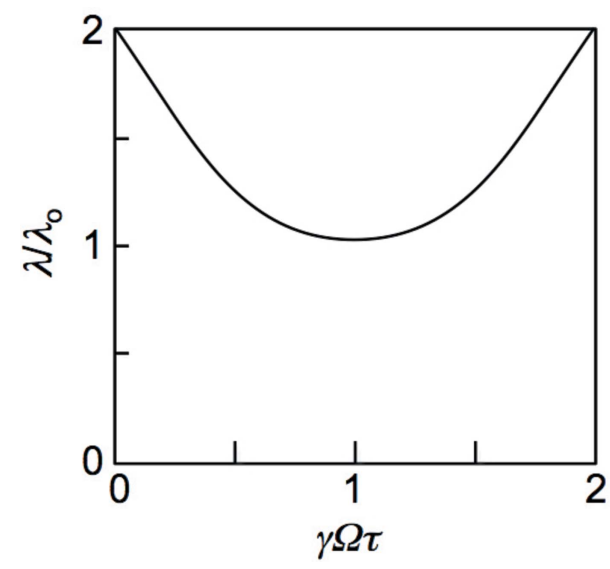

Figure 4

Plot of equation (23), showing how the detected wavelength changes with time. 


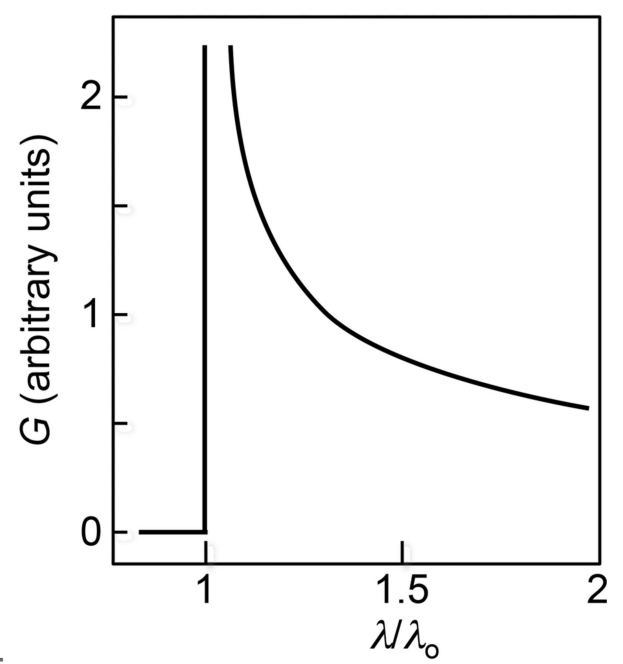

Figure 5

Plot of the spectral distribution of equation (27).

is homogeneous within the emission cone, and zero outside it. Thus, for the detected-wavelength interval from $\lambda_{0}$ to $2 \lambda_{0}$,

$$
\begin{aligned}
G(\lambda) & =P_{0} \frac{\mathrm{d} \tau}{\mathrm{d} \lambda} \\
& = \pm \frac{P_{0}}{2 \gamma \Omega \lambda_{0}} \frac{1}{\left[\left(\lambda / \lambda_{0}\right)-1\right]^{1 / 2}} \\
& =\frac{P_{0}}{2 \gamma \Omega \lambda_{0}} \frac{1}{\left[\left(\lambda / \lambda_{0}\right)-1\right]^{1 / 2}}
\end{aligned}
$$

(the negative solution must be rejected as it is not physically meaningful). Outside the detected-wavelength interval, of course, $G(\lambda)=$ zero.

Fig. 5 shows the plot of $G(\lambda)$. The qualitative correspondence with respect to Fig. 1 is quite evident. We can specifically see the marked asymmetry that corroborates the qualitative justification of the previous section and proves the role of the directional Doppler shift.

However, the lineshape of Fig. 1 is broadened with respect to Fig. 5, and this eliminates in particular the non-physical divergence of equation (27) for $\lambda=\lambda_{0}$. Note that the divergence would be eliminated by a broadening of any origin, and we can propose at least two causes for it: first, the already mentioned Fourier broadening linked to the duration of the detected signal. Second, the fact that the angular power distribution within the emission cone is not homogeneous (as we assumed) but close to Gaussian. This angular distribution affects the spectral lineshape in two different ways. First, it shortens the pulse duration enhancing the Fourier broadening. Second, it modulates the effect of the directional Doppler shift.

Fig. 6 compares the spectrum of Fig. 1 with a broadened version of equation (27) and Fig. 5. The two possible broadening effects were simulated by convolutions with a Gaussian and a half-Gaussian, both with an arbitrarily selected width parameter $\sigma=0.7$, demonstrating that the divergence of equation (27) can indeed be eliminated without removing the asymmetry.

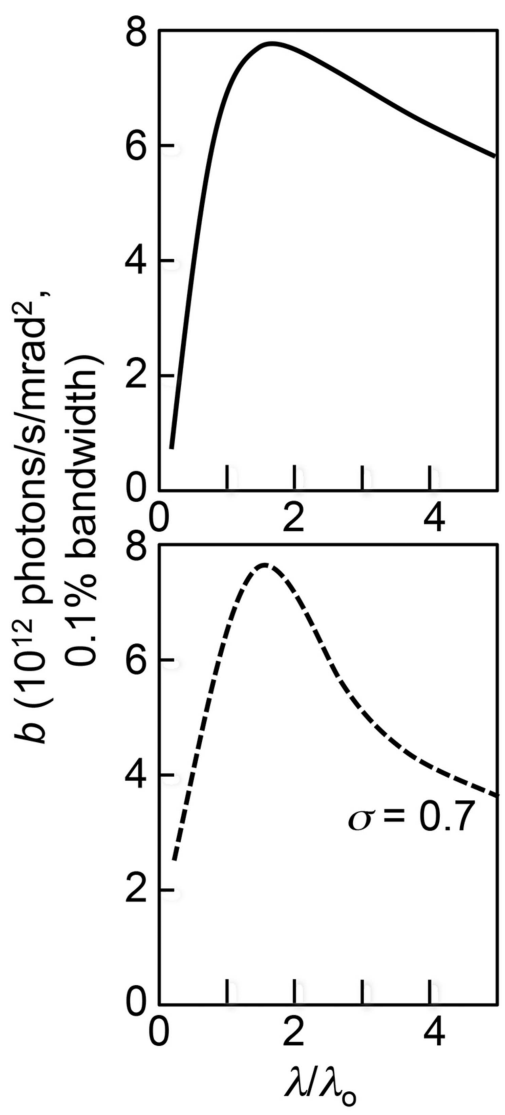

Figure 6

Comparison of the spectrum of Fig. 1 with a doubly broadened version of Fig. 5 (normalized to the top curve).

The comparison of Fig. 6 reveals a reasonable qualitative correspondence but not a close fit. Thus, our approach, as all simplified models, should not be overextended beyond its scope, and certainly cannot replace full theories. We trust, however, that it can be helpful in qualitatively illustrating the role of the directional Doppler shift, not readily evident from previous simplified models.

Before concluding, note that the existing simplified models of undulators (Margaritondo, 2002) do take into account this role. Notably, the emission angular spread is estimated from the fact that the Doppler shift for the maximum angle cannot give photon energies beyond the 'natural' bandwidth, determined by the number of periods in the magnet array.

\section{Funding information}

The following funding is acknowledged: Ecole Polytechnique Fédérale de Lausanne (EPFL) and its Center for Biomedical Imaging (CIBM).

\section{References}

Artsimivich, L. A. \& Pomeranchuk, I. Ya. (1946). J. Exp. Theor. Phys. 16, 379.

Attwood, D. (2007). Soft X-rays and Extreme Ultraviolet Radiation: Principles and Applications. Cambridge University Press. Iwanenko, D. \& Pomeranchuk, I. Ya. (1944). Phys. Rev. 65, 343. 
Margaritondo, G. (1988). Introduction to Synchrotron Radiation. New York: Oxford.

Margaritondo, G. (2002). Elements of Synchrotron Light for Biology, Chemistry, and Medical Research. New York: Oxford.

Margaritondo, G. \& Rafelski, J. (2017). J. Synchrotron Rad. 24, 898901.

Margaritondo, G. \& Rebernik Ribic, P. (2011). J. Synchrotron Rad. 18, 101-108.
Mobilio, S., Boscherini, F. \& Meneghini, C. (2015). Synchrotron Radiation Basics, Methods and Applications. Berlin: Springer.

Pomeranchuk, I. Ya. (1939). J. Exp. Theor. Phys. 9, 915.

Rafelski, J. (2017). Relativity Matters: From Einstein's EMC2 to Laser Particle Acceleration and Quark-Gluon Plasma. Berlin: Springer. Ribic, P. R. \& Margaritondo, G. (2012). J. Phys. D, 45, 213001.

Schwinger, J. (1949). Phys. Rev. 75, 1912-1925. 